\title{
Genome-wide analysis of DNA methylation in rat lungs with lipopolysaccharide-induced acute lung injury
}

\author{
XIAO-QIANG ZHANG ${ }^{1}$, CHANG-JUN LV ${ }^{1}$, XIANG-YONG LIU ${ }^{2}$, DONG HAO ${ }^{1}$ \\ JING QIN $^{1}$, HUAN-HUAN TIAN ${ }^{1}$, YAN LI ${ }^{1}$ and XIAO-ZHI WANG ${ }^{1}$ \\ ${ }^{1}$ Department of Respiratory and Intensive Care Unit, Affiliated Hospital of Binzhou Medical University, Binzhou, \\ Shandong 256603; ${ }^{2}$ Department of Cell Biology, Binzhou Medical University, Yantai, Shandong 264003, P.R. China
}

Received November 13, 2012; Accepted March 7, 2013

DOI: $10.3892 / \mathrm{mmr} .2013 .1405$

\begin{abstract}
Acute lung injury and acute respiratory distress syndrome (ALI/ARDS) are associated with high morbidity and mortality in patients, however, the precise pathogenesis of ALI/ARDS remains unknown. Lipopolysaccharide (LPS) exhibits a number of critical functions and may be associated with the DNA methylation of genes in the lungs. In the present study a genome-wide analysis of DNA methylation was performed in rat lungs with LPS-induced ALI/ARDS. Normal and LPS-induced lung tissues with ALI were analyzed using methylated DNA immunoprecipitation and a rat DNA methylation promoter plus $\mathrm{CpG}$ island microarray and the candidate genes were validated by quantitative reverse transcriptase polymerase chain reaction (qRT-PCR). Aberrant DNA methylation of the promoter regions of 1,721 genes and the $\mathrm{CpG}$ islands of 990 genes was identified when normal lung tissues and lung tissues with LPS-induced ALI/ARDS were compared. These genes were commonly located on chromosomes 1, 3, 5, 7 and 10 ( $\mathrm{P}<0.01)$. Methylation level and $\mathrm{CpG}$ density were compared and it was found that genes associated with high $\mathrm{CpG}$ density promoters had a high ratio of methylation. Furthermore, we performed gene ontology (GO) and Kyoto Encyclopedia of Genes and Genomes (KEGG) pathway analysis. In addition, three genes (Mapk3, Pak1 and Rac2) were validated in the control and lung tissues with ALI by RT-PCR. The results
\end{abstract}

Correspondence to: Professor Xiao-Zhi Wang, Department of Respiratory and Intensive Care Unit, Affiliated Hospital of Binzhou Medical University, 661 Yellow River Road, Binzhou, Shandong 256603, P.R. China

E-mail: respirationbzmc@yahoo.cn

Abbreviations: LPS, lipopolysaccharide; ALI, acute lung injury; ARDS, acute respiratory distress syndrome; PBS, phosphate-buffered saline; HCP, high density $\mathrm{CpG}$ promoters; $\mathrm{LCP}$, low density $\mathrm{CpG}$ promoters; ICP, intermediate density $\mathrm{CpG}$ promoters; MeDIP, methylated DNA immunoprecipitation; GO, gene ontology; KEGG, Kyoto Encyclopedia of Genes and Genomes.

Key words: lipopolysaccharide, acute lung injury, acute respiratory distress syndrome, DNA methylation, promoter, $\mathrm{CpG}$ island indicate that aberrant DNA methylation of lung tissues may be involved in the pathophysiology of LPS-induced ALI/ARDS. Future studies are required to evaluate the therapeutic and prognostic value of the current novel observations in ALI/ARDS.

\section{Introduction}

Acute lung injury (ALI) and its more severe form, acute respiratory distress syndrome (ARDS), characterized by non-cardiogenic pulmonary edema that results from the disruption of the alveolar-capillary barrier and pulmonary capillary permeability, are severe diseases with high clinical morbidity and mortality. Although previous studies have reported a reduction in mortality, due to the implementation of lung-protective ventilation strategies, the mortality rate remains high $(\sim 40 \%)(1,2)$. One of the main pathogenetic factors is sepsis and certain studies have demonstrated that sepsis-induced ALI/ARDS is closely associated with levels of lipopolysaccharide (LPS) in plasma (3). Systemic inflammatory response syndrome (SIRS) is induced by pro- and anti-inflammatory cytokine imbalance and has a detrimental role in LPS-induced ALI/ARDS. LPS causes the simultaneous upregulation or downregulation of the expression of specific inflammatory factors, which leads to changes in the DNA methylation of these factors. LPS induces the hypermethylation of the TNF- $\alpha$ promoter in human THP-1 monocytes. A previous study indicated that epigenetics are significant in the inflammatory process, regardless of whether it occurs locally or systemically (4). Other studies have demonstrated that IL-8 activation in human intestinal epithelial cells is accompanied by $\mathrm{H} 3 \mathrm{~K} 4, \mathrm{H} 3 \mathrm{~K} 9$ and $\mathrm{H} 3 \mathrm{~K} 27$ methylation at the IL-8 gene promoter following LPS stimulation (5). In addition, LPS induces aberrant hypermethylation of Hic-1 in mouse embryonic fibroblasts lacking p53 in culture (6). These findings led us to hypothesize that altered DNA methylation in lung tissues may play a major role in LPS-induced ALI/ARDS.

Epigenetics, including DNA methylation, histone modifications and non-coding RNAs, affect the expression of individual genes, shape developmental patterns and contribute to the maintenance of cellular memory required for developmental stability and tissue-specific changes (7). DNA methylation, as a major form of epigenetic modification, is an important mechanism for the regulation of genome function. DNA methylation 
has a fundamental role in the regulation of gene transcription without altering the sequence of the DNA (8). CpG islands, defined as short DNA regions of genome containing a high frequency of $\mathrm{CG}$ dinucleotides, are often located in the promoter in the 5 ' flanking region of housekeeping genes and a number of tissue-specific genes. Cytosines located at $\mathrm{CpG}$ dinucleotides catalyze this chemical modification and are targeted primarily by the DNA methyltransferase family (9). DNA methylation regulates gene expression by inhibiting the binding of transcription factors to cognate cis elements and by facilitating the binding of methyl-CpG-binding proteins, which directly or indirectly affect the histone code and lead to chromatin condensation to inhibit transcription factor binding (10). In previous studies, the effect of DNA methylation has been associated with cancer, cardiovascular disease, mental illness and human autoimmune diseases. Within the lungs, aberrant DNA methylation is associated with tumorigenesis (11), airway inflammation (12) and other diseases (13).

In the current study, genome-wide analysis of DNA methylation in rat lung tissues with LPS-induced ALI/ARDS was performed using methylated DNA immunoprecipitation (MeDIP) and the Roche-NimbleGen Rat DNA methylation $385 \mathrm{~K} \mathrm{CpG}$ islands plus promoter arrays. Based on results of the MeDIP and arrays, associated genes and chromosomes were determined, the correlation between DNA methylation and $\mathrm{CpG}$ density was determined and gene ontology (GO) and pathway analysis was performed. These results are likely to provide insight into the therapy and prognosis of LPS-induced ALI/ARDS

\section{Materials and methods}

Animals and reagents. Male Sprague-Dawley rats (6-8-weeks old) weighing 180-220 g, were obtained from the Vital River Laboratory Animal Technology Co., Ltd. (Beijing, China). All animals were allowed food and tap water ad libitum. All experimental procedures were in accordance with the Declaration of Helsinki of the World Medical Association. Protocols were also approved by the Institutional Animal Care and Use Committee of Binzhou Medical University. LPS (Escherichia coli LPS, 055:B5) was purchased from Sigma-Aldrich (St. Louis, MO, USA).

LPS-induced ALI animal model. Rats were fasted overnight but allowed water ad libitum prior to induction of ALI. Animals were anesthetized using $40 \mathrm{mg} / \mathrm{kg}$ chloral hydrate. LPS $[10 \mathrm{mg} / \mathrm{kg}$ in phosphate-buffered saline (PBS)] was instilled intratracheally to induce ALI. The control group underwent the same procedure with intratracheal instillation of PBS (10 mg/kg).

Pulmonary histopathology. The lower lobe of the right lung tissue was harvested $12 \mathrm{~h}$ following LPS or PBS administration and fixed in $4 \%$ paraformaldehyde for 5 days at $4^{\circ} \mathrm{C}$. The lobe was embedded in paraffin and cut into $5-\mu \mathrm{m}$ sections. Hematoxylin and eosin staining was performed according to the standard method to assess the lung injury.

Genomic DNA extraction and fragmentation. Genomic DNA was extracted from four lung tissue samples (control and
LPS-induced ALI, $\mathrm{n}=2$ each) using a DNeasy Blood and Tissue kit (Qiagen, Hilden, Germany) according to the manufacturer's instructions. Briefly, the lung tissue was ground using a homogenizer on ice, lysed with proteinase $\mathrm{K}$ and tissue lysis buffer for $3 \mathrm{~h}$ and then precipitated and washed. The genomic DNA quality and quantity was assessed using the Nanodrop spectrophotometer (Thermo Fisher Scientific Inc., Wilmington, DE, USA) and a $\mathrm{A}_{260} / \mathrm{A}_{280}$ ratio between 1.7 and 2.0 was considered a criterion for quality control. The genomic DNA of each sample was sonicated between 200 and 1,000 bp with a Bioruptor sonicator (Diagenode Inc., Denville, NJ, USA) on 'LOW' mode for 10 cycles of $30 \mathrm{sec}$ 'ON' and $30 \mathrm{sec}$ 'OFF'.

MeDIP and microarray analysis. Sonicated genomic DNA $(1 \mu \mathrm{g})$ was used for immunoprecipitation with a mouse monoclonal anti-5-methylcytosine antibody (Diagenode Inc.). DNA was heat-denatured at $94^{\circ} \mathrm{C}$ for $10 \mathrm{~min}$, rapidly cooled on ice and immunoprecipitated with $1 \mu \mathrm{l}$ primary antibody overnight at $4^{\circ} \mathrm{C}$ with rocking agitation in $400 \mu \mathrm{l}$ immunoprecipitation buffer $(0.5 \%$ BSA in PBS). A total of $200 \mu \mathrm{l}$ anti-mouse IgG magnetic beads were added and the mixture was incubated to recover the immunoprecipitated DNA fragments for an additional $2 \mathrm{~h}$ at $4^{\circ} \mathrm{C}$ with agitation. Following immunoprecipitation, five immunoprecipitation washes were performed with ice-cold immunoprecipitation buffer. Washed beads were resuspended in TE buffer with $0.25 \%$ sodium dodecyl sulfate (SDS) and $0.25 \mathrm{mg} / \mathrm{ml}$ proteinase $\mathrm{K}$ for $2 \mathrm{~h}$ at $65^{\circ} \mathrm{C}$ and then allowed to cool to room temperature. MeDIP DNA was purified using Qiagen MinElute columns (Qiagen). MeDIP-enriched DNA was amplified using the GenomePlex® Complete Whole Genome Amplification kit from Sigma-Aldrich. Amplified DNA samples were purified using the QIAquick PCR purification kit (Qiagen). Purified DNA was quantified using the ND-1000 Nanodrop. For DNA labeling, the NimbleGen Dual-Color DNA Labeling kit was used according to the manufacturer's instructions (NimbleGen Systems, Inc., Madison, WI, USA). The DNA $(1 \mu \mathrm{g})$ of each sample was incubated for $10 \mathrm{~min}$ at $98^{\circ} \mathrm{C}$ with $1 \mathrm{OD}$ or $40 \mu \mathrm{l}$ of Cy5-9mer (MeD1P sample) or Cy3-9mer (input sample) primers. Next, 100 pmol deoxynucleoside triphosphates and 100 units Klenow fragment (New England Biolabs, Inc., Ipswich, MA, USA) were added. The mix was incubated at $37^{\circ} \mathrm{C}$ for $2 \mathrm{~h}$. The reaction was terminated by adding $10 \mu \mathrm{l} 0.5 \mathrm{M}$ EDTA and the labeled DNA was purified by isopropanol/ethanol precipitation. Microarrays were hybridized at $42^{\circ} \mathrm{C}$ for $16-20 \mathrm{~h}$ with $\mathrm{Cy} 3 / 5$-labeled DNA in NimbleGen hybridization buffer/hybridization component A in a hybridization chamber (Hybridization System, NimbleGen Systems). Following hybridization, washing was performed using the NimbleGen Wash Buffer kit. For array hybridization, Roche-NimbleGen's Rat Promoter plus CpG Island array was used. The array has a $385 \mathrm{~K}$ format array design containing gene promoters $[-1,300$ to $+500 \mathrm{bp}$ of the start site of the transcript (TSS)]. A total of 15,809 CpG islands were covered by $\sim 385,000$ probes. Array data were extracted and analyzed using NimbleScan and SignalMap software. Only genes with consistent differences between the two control and two LPS groups were considered.

Quantitative reverse transcription-polymerase chain reaction (qRT-PCR) confirmation of gene methylation changes. A 

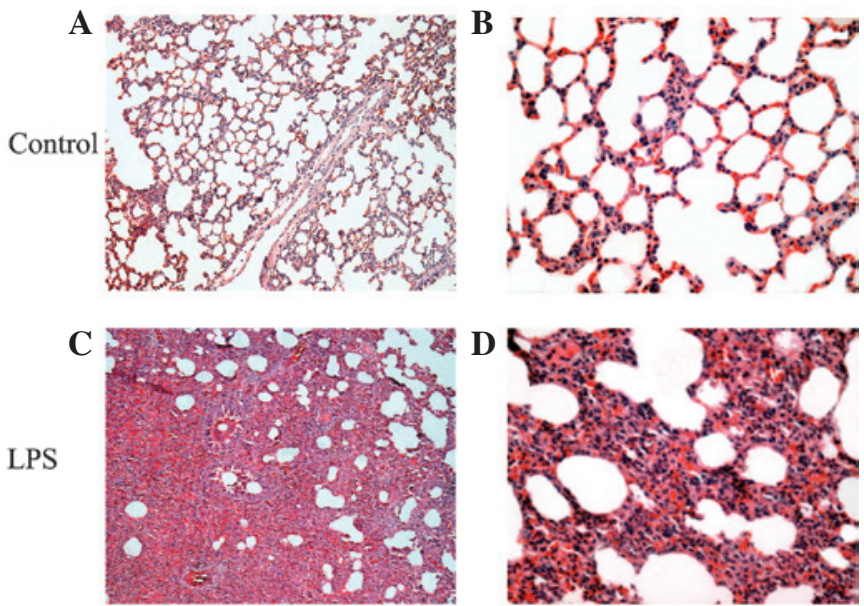

Figure 1. Pulmonary histopathological changes in LPS-administered rats Lung tissue specimens were obtained from the control group, magnification (A) x100 and (B) x400; and LPS group, magnification (C) x100 and (D) x400. LPS, lipopolysaccharide.

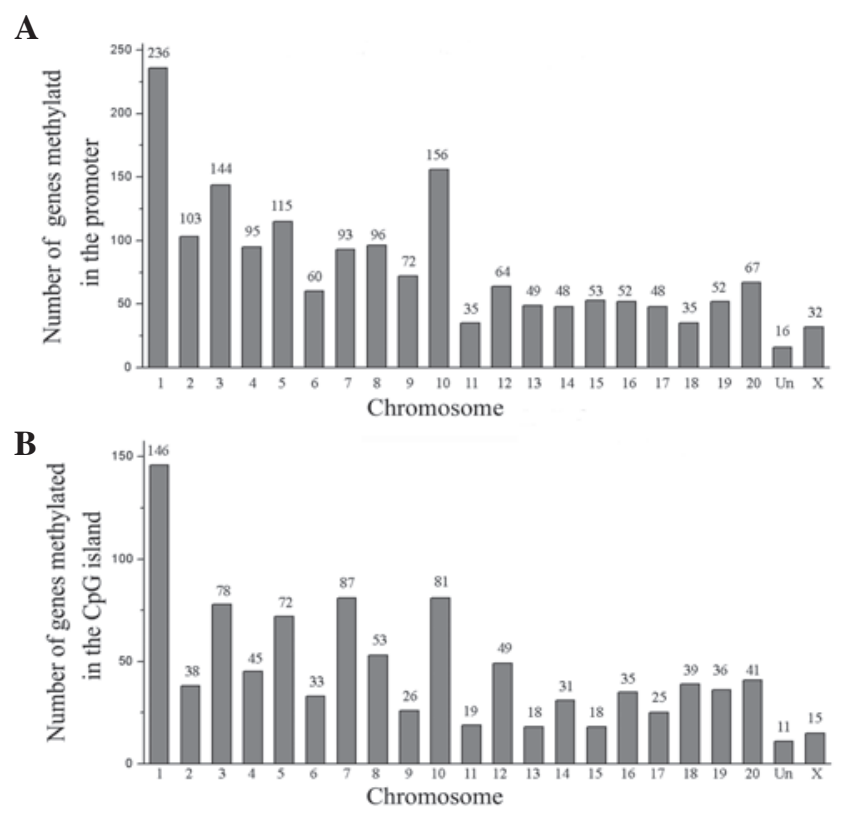

Figure 2. Number of genes methylated in the promoter and $\mathrm{CpG}$ island. (A) A total of 1,721 candidate genes in the promoter were distributed across all chromosomes. (B) 990 candidate genes in the $\mathrm{CpG}$ island were distributed across all chromosomes.

MeDIP assay, combined with qPCR, was used to quantitatively evaluate the methylation status of candidate genes in the lung tissues derived from control and ALI/ARDS groups. MeDIP was performed as described. Purified DNA from the immunoprecipitated DNA complexes and the input DNA was analyzed by qRT-PCR on the ABI PRISM 7900 system (Applied Biosystems, Bedford, MA, USA). Primers used were as follows: Mapk3, forward 5'-CCCTTCAGACTGCTTCCTCA-3' and reverse 5'-CTT GGGCTGTCAGACTTGGT-3'; Pak1, forward 5'-GAATTT GTGGTACAGCAGGACAT-3' and reverse 5'-CCACTGAGG CTATCTTTGACG-3'; Rac2, forward 5'-TTACCCATCACC CACCACC-3' and reverse 5'-TTCCGTTTCCTCCTGCCTC-3'. Relative changes in gene methylation were determined by measuring the amount of detected genes in immunoprecipitated DNA following normalization against input DNA.

Statistical analysis. Data are expressed as the mean \pm SD. For the chromosome distribution of genes and the number of genes in high $\mathrm{CpG}$ density promoters (HCP), intermediate $\mathrm{CpG}$ density promoters (ICP) and low $\mathrm{CpG}$ density promoters (LCP), positive/negative genes were compared using the Chi-square test. $\mathrm{P}<0.05$ was considered to indicate a statistically significant difference (14).

\section{Results}

Histological changes in lung tissues. No histological alterations were found in the control group (Fig. 1A and B). In the LPS-induced ALI/ARDS group, microscopic changes were observed $12 \mathrm{~h}$ following LPS administration. The observed inflammatory alterations were characterized by alveolar wall thickness, alveolar and interstitial edema and hemorrhage, interstitial infiltration by neutrophils and the complete consolidation of a section of the lung tissue (Fig. 1C and D).

Chromosomal distribution. LPS-induced DNA methylation alterations were initially observed in the chromosome. A total of 1,721 candidate genes methylated in the promoter region were distributed across all chromosomes (Fig. 2A). The results indicate that the gene number of the chromosomes was statistically significant: 236 genes on chromosome 1 (13.7\%, $\mathrm{P}<0.01), 144$ genes on chromosome 3 (8.4\%, $\mathrm{P}<0.01)$, 115 genes on chromosome $5(6.7 \%, \mathrm{P}<0.01)$ and 156 genes on chromosome $10(9.1 \%, \mathrm{P}<0.01)$. The 990 candidate genes methylated in the $\mathrm{CpG}$ island were also distributed across all chromosomes (Fig. 2B). Results indicate that the gene number of the chromosomes was statistically significant: 146 genes on chromosome $1(14.7 \%, \mathrm{P}<0.01), 78$ genes on chromosome 3 (7.9\%, $\mathrm{P}<0.01), 72$ genes on chromosome $5(7.3 \%, \mathrm{P}<0.01)$, 81 genes on chromosome $7(8.2 \%, \mathrm{P}<0.01)$ and 81 genes on chromosome $10(8.2 \%, \mathrm{P}<0.01)$.

Levels of DNA methylation in the promoters and CpG islands. The promoters were divided into three categories based on CG content: HCP, ICP and LCP. The methylation level and CpG density in the promoter were compared (Fig. 3A). In the group of genes in which the degree of methylation was reduced by LPS-induced ALI/ARDs, the number of methylated HCP genes was significantly higher $(49.34 \%, \mathrm{P}<0.01)$ than the numbers of methylated ICP (26.11\%) and LCP $(24.56 \%)$ genes. However, in the group of genes in which the degree of methylation was increased by LPS-induced ALI/ ARDs, the numbers of methylated genes were similar in the HCP $(32.39 \%)$, ICP (32.15\%) and LCP (35.46\%) zones. In addition, differences in the methylation levels of $\mathrm{CpG}$ islands were noted (Fig. 3B). In the decreased group, the number of methylated HCP genes was also significantly higher $(59.18 \%$, $\mathrm{P}<0.01)$ than that of ICP $(38.78 \%)$ and LCP $(2.04 \%)$ genes. A similar distribution was observed in the increased group: the methylation of the HCP zone $(60.26 \%)$ was identified to be significantly higher $(\mathrm{P}<0.01)$ than that of the ICP $(26.67 \%)$ and LCP (13.07\%) zones. 
Table I. GO annotation of the candidate genes identified by microarray. Methylated genes involved in the response to external stimulus, intracellular signal transduction and negative regulation of cell proliferation.

GO term Focus genes Gene name

Intracellular

signal transduction

Negative regulation of cell proliferation

Response to external 146 stimulus

205

\begin{abstract}
ADRA2B//PAK1//MAPK3//MAP2K2//TGFB1//MAPK12//RGD1562846//CDKN1A// FOXM1//HTR6//PTGER3//GCGR//CNR1//GNAZ//MC3R//GHRH//AVPR1B// GLP2R//PTHLH//GNAS//ADRB1//ADORA2A//GALR1//ADCY5//DRD3//NPR3// INSL3//GRM7//GRIK3//FZD1//NMUR1//CASR//ATP2B4//LAT//RCAN2//ALMS1// RCAN3//SIK1//MARK2//SOCS3//MAP4K2//SRPK2//STK4//RPS6KA5//MAST1// CARD9//CSNK2B//SNIP1//AZI2//RIPK2//AGT//ERC1//TRIB1//ROR2//STRADB// DAB2IP//WNT7B//GAB1//MAPK8IP1//GH1//F2R//MRAS//RAB4B//ARL3// RAB6A//RHOQ//ARHGEF7//RALB//ARFRP1//RAB35//ARL9//RAB40B// RHOBTB1//RASL12//RAB40C//DNAJA3//RGD1307615//REM1//DIRAS1//RAC2// RAB20//RAB1B//GRB2//SYNGAP1//RASSF1//RSU1//CDC42EP1//ARHGDIA// XPA//PDE4D//MIF//FGF1//PIK3R1//PLEKHA1//LIME1//RELN//TGFB2//PTPN6// GPER//UBE3A//TBXA2R//PDE7A//IGFBP1//FOXO3//RPS6KB1//EIF4EBP1// DISC1//HIF1A//AKT1S1//TMEM127//CYTH1//IQSEC3//CYTH4//GSTP1//CRHR2// TAOK1//STMN3//ARHGEF15//ARHGEF3//FARP1//PLEKHG4//RGNEF//NGFR// LPAR1//LPAR2//SOX11//PTK6//IL6//IL3//PHLDA3//PDPK1//NUP62//SLC20A1// ATP2C1//TRADD//CXXC5//UBE2V1//LTBR//MAVS//NEK6//GOLT1B//MYLK2// P2RX7//PTPRC//INSR//FGFR2//LPAR3//FGFR1//FLT4//IGFBP4//AKT2//RPS6KB2// MAP3K10//FZD5//LOC682999//SNAI1//CDC34//MECOM//SERPINF2//CD27// LEPROT//RASA2//TNK1//CMKLR1//ADA//VEGFB//F7//TCF7L2//CCL11//DUSP6// NDRG2//FGF21//ARHGAP8//MYBBP1A//CASP3//RGS7//ECEL1//UNC13A// PDZD2//GUCY2E//SMAD7//CSPG4//PSEN2//DUSP1//PBP2//PLCL2//SPSB3// ADCY1//PDZD8//ARHGAP29//HMHA1//PLCZ1//ASB10//SOCS5
\end{abstract}

SULF1//KRIT1//TGFB1//ASCL2//CASP3//DLG1//SCGB1A1//PTPN6//GSTP1//LTA// DAB2IP//APOD//PIK3R1//TRIB1//SF1//NDRG2//ANG1//GAL//LST1//SOX11// FGFR2//GPC3//TGFB2//PTCH1//RUNX3//LRP6//KRT4//PAK1//TSPO//GATA2// WT1//AGT//CEBPA//IL6//JUN//NOS1//FOXA3//BDKRB2//PHB//ADORA2A//F2R// PPARG//BMP2//WISP2//PTGES//NUP62//GABBR1//INSL3//BECN1//CDKN1A// ALDH1A2//PTPRU//TFF1//SOX7//ENPP7//ROR2//CDH5//TMEM127//STK4// FZD5//PTPRF//DNAJA3//IRF6//LEFTY1//KLF13

BECN1//WIPI2//MAP1LC3A//ATG9A//CLN3//TRPM4//CCL11//PPARG//IL6// ALOX5AP//MIF//F7//CNR1//LTA//CMKLR1//CXCL2//S100A8//CCR10//RAC2// JUN//JUND//RELN//MGP//ACCN1//KCNA5//BMP2//HIF1A//P2RX7//CTSB// RPS6KB1//PTCH1//ACCN3//NGFR//NKX2-1//PLA2G10//APBB1//SEMA6C// SEMA3A//MYH10//TGFB2//EFNA2//NFASC//RUNX3//GDF7//EPHB3//PGRMC1// KLF7//NRCAM//CEBPA//TBXA2R//PIK3R1//COX4I1//DDIT3//PLEC//CHAT// SLC6A19//OPN4//TULP1//PDE6C//HOXA2//FOXA3//CARTPT//GAS2L1//PDE6B// GRK1//TSPO//RARRES2//FOXG1//AGT//MAPK3//BAD//CYBA//BNIP3//LTBR// HABP4//F2R//CR1L//ZIC2//ACE//GSTP1//LIPC//NOS1//INSR//SDS//TH//AKT2// THRA//SLC1A2//CDKN1A//PRDM4//G6PD//GH1//CLPS//GHRH//GHSR//SOCS3// PNLIPRP2//SLC22A3//IGF2R//PTGES//SCAMP3//RPL36AL//DUSP1//DKK1// CLK2//LEFTY1//PITX2//ALDH1A2//ADA//ORM1//HMGCS1//ALPL//TGFB1// TRIM25//EGR3//MYBBP1A//PFKFB1//AK3//ACADS//SSTR3//AANAT//DSCAM// FOXE1//SCGB1A1//SETD6//ADORA2A//CNR2//HSPD1//DRD3//ATP2B2//CDH23// FGF7//VEGFB//ZFP354A//LRP6//SERPINF2//LTC4S//PTK7//WNT7B//RXRB// PTK6//FZD1//SKP2//LPAR1//MECOM//RPS6KA5
GO annotation and pathway analysis. The GO project provides a controlled vocabulary to describe gene and gene product attributes in any organism (http://www.geneontology.org). The ontology covers three domains: biological process, cellular component and molecular function. In the present study, GO Ontology was used to perform GO term analysis of the 
Table II. Methylated gene association studies in acute lung injury and acute respiratory distress syndrome (ALI/ARDS).

\begin{tabular}{|c|c|c|}
\hline Gene symbol & Protein name & Description \\
\hline Ace & $\begin{array}{l}\text { Angiotensin-converting } \\
\text { enzyme }\end{array}$ & $\begin{array}{l}\text { Catalyzes the conversion of angiotensin I to angiotensin; plays a role in } \\
\text { regulation of blood pressure. }\end{array}$ \\
\hline Akt2 & $\begin{array}{l}\text { RAC }-\beta \text { serine/threonine } \\
\text { protein kinase }\end{array}$ & Involved in phosphatidylinositol 3-kinase-mediated signaling. \\
\hline Casp3 & Caspase-3 & $\begin{array}{l}\text { Apoptotic cysteine-aspartic acid protease that may play a role in neuronal cell } \\
\text { death regulation and other apoptotic processes. }\end{array}$ \\
\hline Cebpb & $\begin{array}{l}\text { CCAAT/enhancer-binding } \\
\text { protein } \beta\end{array}$ & $\begin{array}{l}\text { Transcription factor that binds to CCAAT motif on DNA and may facilitate IL-6 } \\
\text { induced transcriptional activation. }\end{array}$ \\
\hline $\mathrm{Cxcl} 2$ & C-X-C motif chemokine 2 & Chemokine involved in the pulmonary inflammatory response. \\
\hline IL6 & Interleukin-6 & Cytokine involved in development and possibly in neurodegenerative processes. \\
\hline Mapk3 & $\begin{array}{l}\text { Mitogen-activated protein } \\
\text { kinase } 3\end{array}$ & $\begin{array}{l}\text { Kinase involved in intracellular signalling; component of MAPK signaling } \\
\text { pathway. }\end{array}$ \\
\hline Mif & $\begin{array}{l}\text { Macrophage migration } \\
\text { inhibitory factor }\end{array}$ & $\begin{array}{l}\text { Inhibits random migration of macrophages and is involved in the pathogenesis } \\
\text { of several inflammatory diseases. }\end{array}$ \\
\hline Mylk2 & $\begin{array}{l}\text { Myosin light chain kinase } \\
2, \text { skeletal/cardiac }\end{array}$ & Kinase; phosphorylates a serine in the $\mathrm{N}$-terminus of a myosin light chain. \\
\hline Pak1 & $\begin{array}{l}\text { Serine/threonine-protein } \\
\text { kinase PAK } 1\end{array}$ & $\begin{array}{l}\text { Serine/threonine protein kinase; binds and complexes specifically with } \\
\text { activated (GTP-bound) p } 21 \text {, leading to inhibition of p } 21 \text { GTPase activity. }\end{array}$ \\
\hline Rac2 & $\begin{array}{l}\text { Ras-related C3 botulinum } \\
\text { toxin substrate } 2\end{array}$ & $\begin{array}{l}\text { Exhibits GTPAse activity, protein binding (homolog); involved in actin } \\
\text { cytoskeleton organization and biogenesis, bone resorption; associated with } \\
\text { neutrophil immunodeficiency syndrome. }\end{array}$ \\
\hline Tgfb1 & $\begin{array}{l}\text { Transforming growth } \\
\text { factor } \beta-1\end{array}$ & $\begin{array}{l}\text { Binds the TGF } \beta \text { receptor; plays a role in regulation of cell growth and } \\
\text { proliferation; induces synthesis of extracellular matrix proteins and may play } \\
\text { a role in fibrosis. }\end{array}$ \\
\hline Tgfb2 & $\begin{array}{l}\text { Transforming growth } \\
\text { factor } \beta-2\end{array}$ & $\begin{array}{l}\text { Binds the transforming growth factor- } \beta \text { receptor; plays a role in regulation of } \\
\text { cell growth and proliferation; may be involved in mesenchymal-epithelial cell } \\
\text { interactions during development. }\end{array}$ \\
\hline Vegfb & $\begin{array}{l}\text { Vascular endothelial } \\
\text { growth factor B }\end{array}$ & Mouse homolog is a growth factor; involved in the promotion of angiogenesis. \\
\hline
\end{tabular}

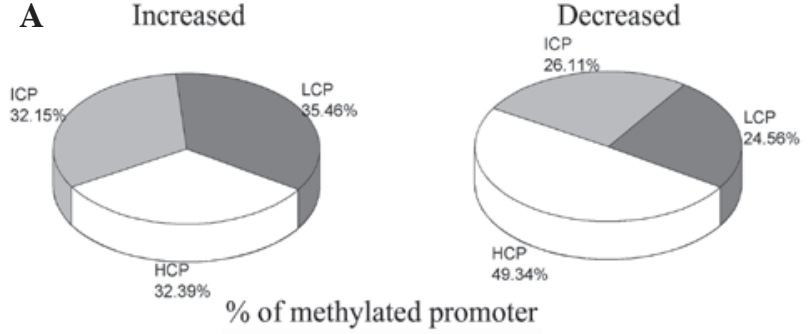

B Increased

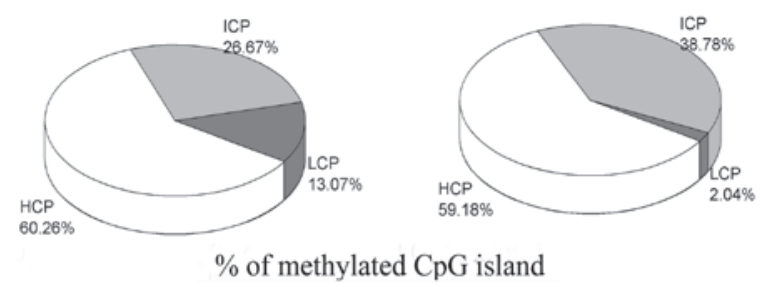

Figure 3. Levels of DNA methylation in the promoter and $\mathrm{CpG}$ island. Methylation level and the $\mathrm{CpG}$ density in the (A) promoter and (B) $\mathrm{CpG}$ island. $\mathrm{HCP}$, high $\mathrm{CpG}$ density promoter; ICP, intermediate $\mathrm{CpG}$ density promoter; $\mathrm{LCP}$, low $\mathrm{CpG}$ density promoter.
1,721 genes in the methylated in the promoter region and the 990 genes methylated in the $\mathrm{CpG}$ island. Results indicate that the candidate genes are associated with 755 biological processes, 79 cellular components and 93 molecular functions. Genetic studies of ALI/ARDS have largely focused on candidate genes involved in the response to external stimulus, intracellular signal transduction and negative regulation of cell proliferation (15). Therefore, all genes in the three GO terms were selected (Table I). GO analysis of all candidate genes revealed 146 genes involved in the response to external stimulus, 205 genes in intracellular signal transduction and 65 genes in negative regulation of cell proliferation. Next, 14 methylated genes from the GO term results which are etiologically involved in the LPS-induced ALI/ARDS were selected (Table II). The Kyoto Encyclopedia of Genes and Genomes (KEGG) pathway database (http://www. genome.jp/kegg) was used to perform pathway analysis of these candidate genes. The analysis divided the candidate genes into 38 signaling pathways, and the 10 enrichment pathways involved in immune and inflammatory responses were selected. 
Table III. Pathway analysis of the candidate genes identified by microarray.

\begin{tabular}{lcl} 
Signaling pathway & Focus genes & \multicolumn{1}{c}{ Gene name } \\
\hline $\begin{array}{l}\text { Neuroactive ligand } \\
\text { receptor interaction }\end{array}$ & 55 & ADORA2A//ADRA1D//ADRA2B//ADRB1//APLNR//AVPR1B//BDKRB2// \\
& & CHRM1//CHRM5//CHRNA4//CNR1//CNR2//CRHR2//DRD3//F2R//GABBR1// \\
& GABRA3//GABRG3//GABRR3//GALR1//GCGR//GH1//GHSR//GLP2R// \\
& GPR35//GRIA2//GRIK3//GRIK4//GRM7//HRH3//HTR1D//HTR6//LPAR1// \\
& LPAR2//LPAR3//MC3R//MC5R//NMUR1//NTSR1//P2RX7//P2RY14//PPYR1// \\
& PTGER3//PTGIR//SCTR//SSTR3//TAAR1//TAAR3//TAAR4//TAAR6//TAAR9// \\
& TBXA2R//THRA//TSPO//UTS2R
\end{tabular}

Neurotrophin

signaling pathway

MAPK signaling pathway

mTOR signaling

pathway

Fc $\gamma$ R-mediated

phagocytosis

Regulation of actin

cytoskeleton
VEGF signaling pathway

B cell receptor signaling pathway $\mathrm{T}$ cell receptor signaling pathway
AKT2//AKT3//ARHGDIA//BAD//CALML3//CAMK2G//FOXO3//GAB1// GRB2//IRAK2//JUN//MAP2K2//MAPK12//MAPK3//NFKBIE//NGFR//NTF4// PIK3R1//PIK3R2//PRDM4//PSEN2//RIPK2//RPS6KA5//YWHAG//YWHAH

AKT2//AKT3//CACNB2//CACNG1//CACNG5//CACNG6//CACNG8//CASP3// CHP2//DAXX//DDIT3//DUSP1//DUSP14//DUSP6//FGF1//FGF21//FGF7// FGFR1//FGFR2//GRB2//JUN//JUND//MAP2K2//MAP4K2//MAPK12//MAPK3// MAPK8IP1//MECOM//MRAS//NTF4//PAK1//PLA2G10//PLA2G2C// PLA2G2F//PPP3R2//RAC2//RASA2//RPS6KA5//STK4//TAOK1//TGFB1// TGFB2

ADCY1//ADCY5//AKT2//AKT3//CAMK2G//CHAT//CHRM1//CHRM5//
CHRNA4//CREB3L3//GNB3//GNG7//GNG8//KCNJ2//KCNJ3//KCNJ4//
KCNJ6//MAPK3//PIK3R1//PIK3R2//SLC18A3

AKT2//AKT3//EIF4E//EIF4EBP1//HIF1A//MAPK3//PDPK1//PIK3R1//PIK3R2// RPS6KB1//RPS6KB2//VEGFB

AKT2//AKT3//AMPH//ARPC1B//ARPC2//ARPC4//DNM3//FCGR2A//LAT// LIMK1//MAPK3//PAK1//PIK3R1//PIK3R2//PIP5K1B//PTPRC//RAC2// RPS6KB1//RPS6KB2

ACTB//APC2//ARHGEF7//ARPC1B//ARPC2//ARPC4//BDKRB2//CHRM1// CHRM5//F2R//FGD1//FGF1//FGF21//FGF7//FGFR1//FGFR2//ITGB4//LIMK1// MAP2K2//MAPK3//MRAS//MYH10//MYL7//MYLK2//NCKAP1//PAK1// PIK3R1//PIK3R2//PIP4K2B//PIP5K1B//PPP1CA//PPP1R12A//RAC2

AKT2//AKT3//BAD//CHP2//MAP2K2//MAPK12//MAPK3//PIK3R1//PIK3R2// PLA2G10//PLA2G2C//PLA2G2F//PPP3R2//RAC2

AKT2//AKT3//CHP2//DAPP1//GRB2//JUN//MAP2K2//MAPK3//NFKBIE// PIK3AP1//PIK3R1//PIK3R2//PPP3R2//PTPN6//RAC2

AKT2//AKT3//CDK4//CHP2//DLG1//GRB2//JUN//LAT//MAP2K2//MAPK12// MAPK3//NFKBIE//PAK1//PIK3R1//PIK3R2//PPP3R2//PTPN6//PTPRC
The included pathways were: neuroactive ligand-receptor interaction, neurotrophin signaling pathway, MAPK signaling pathway, cholinergic synapse, mTOR signaling pathway, Fc $\gamma$ R-mediated phagocytosis, regulation of actin cytoskeleton, vascular endothelial growth factor (VEGF) signaling pathway, $\mathrm{B}$ cell receptor signaling pathway and $\mathrm{T}$ cell receptor signaling pathway (Table III).

qRT-PCR validation of differential genes in the microarrays. A subset of 3 genes, Mapk3, Pak1 and Rac2, that reveal differential methylation between the control and ALI/ARDS groups were validated using qRT-PCR to confirm the microarray results independently. Mapk3 and Pak1 showed DNA methylation in the control group. However, in the ALI/ARDS group, Rac2 was methylated. A close correlation was observed between the microarray and qRT-PCR data (Table IV), indicating the accuracy of our microarray data and the significant induction in the expression of candidate genes following LPS.

\section{Discussion}

The present study reports, to the best of our knowledge, the first genome-wide DNA methylation analysis of rat lung tissues with LPS-induced ALI/ARDS. A genome-wide DNA methylation analysis of lung tissues with ALI/ARDS was performed in rats. In addition, the promoter regions of 1,721 genes and the $\mathrm{CpG}$ islands of 990 genes were found to exhibit aberrant levels of DNA methylation compared with normal lung tissues. Next, the DNA methylation status of three candidate genes, Mapk3, Pak1 and Rac2, was validated using qRT-PCR. 
Table IV. Gene methylation changes determined by quantitative reverse transcription-polymerase chain reaction.

\begin{tabular}{llccc}
\hline Gene & Sample & Input $(\mathrm{Ct})$ & IP $(\mathrm{Ct})$ & $\%$ \\
\hline Mapk3 & Control & 23.757 & 27.986 & 1.066 \\
& LPS & 24.141 & NA & NA \\
Pak1 & Control & 23.693 & 27.854 & 1.117 \\
& LPS & 23.628 & 39.023 & $4.64 \mathrm{E}-04$ \\
Rac2 & Control & 20.783 & 36.298 & $4.27 \mathrm{E}-04$ \\
& LPS & 20.717 & 25.101 & 0.958 \\
\hline
\end{tabular}

$\%$ input $=2 \times\left(\mathrm{Ct}_{\text {Input }}-\mathrm{Ct}_{\mathrm{ChIP}}\right) \times \mathrm{Fd} \times 100 . \mathrm{Fd}$, input dilution factor; LPS, lipopolysaccharide.

The chromosomal locations of these genes were identified and chromosomes $1,3,5,7$ and 10 were identified to be the most common locations of these genes. Specific genes on these chromosomes, including Mapk3 (16) and Lat (17) on chromosome 1, Mylk2 and Cebpb (17) on chromosome 3, Rac2 (18) on chromosome 7 and Ace (19) on chromosome 10, have been reported to be critical factors in the development of ALI/ARDS. Therefore, aberrant DNA methylation on chromosomes 1,3,5, 7 and 10 may be associated with the pathogenesis of ALI/ARDS.

In the current study, the differences in DNA methylation patterns for 3 classes of $\mathrm{CpG}$ island, HCP, ICP and LCP, were observed. Among the $\mathrm{CpG}$ island distribution categories, a number of genes in HCP may be associated with housekeeping genes and regulate developmental genes, whereas genes in LCP are largely associated with tissue-specific genes (20), which indicates that, based on $\mathrm{CpG}$ density, analyzing methylation changes may provide additional insight. DNA methylation levels differed significantly among the 3 categories. The 1,721 genes methylated in the promoter region include 452 genes with a decreased degree of methylation and 1,269 genes with an increased degree of methylation. The incidence of methylated HCP genes in the decreased group was higher $(\mathrm{n}=223, \mathrm{P}<0.01)$. However, in the increased group, the incidence of methylated genes in the three categories was not found to be significant. A similar distribution was observed in the genes methylated in the $\mathrm{CpG}$ island: methylated HCP genes in the decreased and increased groups were markedly higher $(\mathrm{P}<0.01)$. Results indicate that a higher number of housekeeping and developmental genes are regulated than tissue-specific genes in the pathophysiology of ALI/ARDS. Overall, the observations of the current study demonstrated that DNA methylation is associated with $\mathrm{CpG}$ density, DNA methylation has a higher incidence in HCP genes compared with ICP and LCP genes and housekeeping and developmental genes may play crucial roles in the pathophysiology of LPS-induced ALI/ARDS.

From our methylated genes, which were association studies with positive findings in ALI/ARDS, we identified 14 methylated genes. Among these genes, a substantial number have been demonstrated to play a functional role in LPS-induced ALI/ARDS. Of the 14 methylated genes, angiotensin-converting enzyme (ACE), is the key enzyme that converts AT-I to AT-II and its functions are involved in the positive regulation of apoptotic process, angiotensin signaling process, the renin-angiotensin cascade pathway and angiotensin II signaling pathway. ACE I/D polymorphism affects the prognosis of ALI/ARDS (21). ALI/ARDS is characterized by alveolar injury and increased pulmonary vascular permeability. Mura et al (22) reported a potential role for VEGF in promoting the repair of the alveolar-capillary membrane during recovery from ALI/ARDS. Vegfb is associated with the VEGF signaling pathway and is involved in the promotion of angiogenesis. The methylation of Vegfb may affect repair of the alveolar-capillary membrane and angiogenesis.

One of the principal mechanisms of LPS-induced ALI/ARDS relates to the effects of the inflammatory response, which leads to SIRS, including activation of leukocytes-alveolar macrophages and sequestered neutrophils in the lungs. A previous genetic study on ALI/ARDS reported that genes associated with the inflammatory response are important in the development of ALI/ARDS. The present study found that following genes associated with the inflammatory response exhibited aberrant DNA methylation profiles: i) Cebpb, CCAAT/enhancer-binding protein $\beta$, is a critical regulator of the inflammatory responses and injury in the lungs (23); ii) $\mathrm{Cxcl}_{2}$ is a potent neutrophil chemokine involved in the pulmonary inflammatory response, which is linked to ventilator-induced ALI and hyperoxia-induced ALI. Inhibition of its receptor leads to a marked decrease in neutrophil sequestration and lung injury (24); iii) IL6 is a potent proinflammatory cytokine and key factor in the development of ALI/ARDS (25); iv) Mylk2 encodes proteins involved in multiple components of the inflammatory response, including apoptosis, vascular permeability and leukocyte diapedesis. Myosin light-chain kinase, a central cytoskeletal regulator encoded by Mylk, has a key pathophysiological role in ALI (26); and v) Mif, macrophage migration inhibitory factor, is involved in the pathogenesis of several inflammatory diseases. Mif-induced neutrophils accumulate in the alveolar space, indicating that Mif may be a useful target in the reduction of neutrophil lung inflammation and ALI (27). These methylated genes are involved in important mechanisms that underlie ALI/ARDS. However, further studies are required to identify the correlation between the aberrant methylation of these genes and the pathogenesis of LPS-induced ALI/ARDS.

According to KEGG pathway analysis, 10 enrichment pathways were selected. Of the top 10 enrichment pathways, MAPK is an important signal transmitter from the cell surface to the internal nucleus and is mainly involved in cell differentiation and proliferation, apoptosis and regulation of immune and inflammatory responses. MAPK initiates a cascade of inflammatory cytokines, leading to an uncontrolled inflammatory response. LPS induces an inflammatory reaction through the activation of the MAPK signaling pathway. Thus, the MAPK signaling pathway may have an essential role in the development of pulmonary inflammation and LPS-induced ALI/ARDS. A total of 42 methylated genes are associated with the MAPK signaling pathway and 7 have been associated with ALI/ARDS in previous studies, including Akt2 (28), Casp3 (29), Mapk3 (16), Pak1 (30), Rac2 (18), Tgfb1 and Tgfb2 (31). These genes have a functional role in the MAPK signaling pathway and aberrant methylation of these genes may affect its activation and inflammatory response in LPS-induced ALI/ARDS. 
In summary, the Roche-NimbleGen Rat DNA methylation $385 \mathrm{~K} \mathrm{CpG}$ islands plus promoter array is a useful tool for studying the genome-wide DNA methylation of lung tissues with LPS-induced ALI/ARDS. Aberrant DNA methylation in ALI/ARDS was determined and altered patterns of lung DNA methylation during the pathophysiology of LPS-induced ALI/ARDS were observed. The identification of a lung gene-specific methylation profile may provide valuable insight into pathways that are likely to be epigenetically regulated. Further analysis of DNA methylation is important for the understanding of ALI/ARDS and may be of value for indicating prognostic biomarkers and predictors of response to therapy and may constitute future therapeutic targets.

\section{Acknowledgements}

This study was supported by grants from the Natural Science Foundation of Shandong Province, China (Y2008C163). The authors thank Kangchen Biotech Company for assistance with the analyses.

\section{References}

1. Rubenfeld GD, Caldwell E, Peabody E, et al: Incidence and outcomes of acute lung injury. N Engl J Med 353: 1685-1693, 2005.

2. Zambon M and Vincent JL: Mortality rates for patients with acute lung injury/ARDS have decreased over time. Chest 133 : $1120-1127,2008$.

3. Wang HM, Bodenstein M and Markstaller K: Overview of the patolgy of three widely used animal models of acute lung injury. Eur Surg Res 40: 305-316, 2008.

4. El Gazzar M, Yoza BK, Hu JY, Cousart SL and McCall CE: Epigenetic silencing of tumor necrosis factor alpha during endotoxin tolerance. J Biol Chem 282: 26857-26864, 2007.

5. Angrisano T, Pero R, Peluso S, et al: LPS-induced IL-8 activation in human intestinal epithelial cells is accompanied by specific histone $\mathrm{H} 3$ acetylation and methylation changes. BMC Microbiol 10: 172, 2010.

6. Tatemichi $M$, Hata $H$, Tazawa $H$ and Nakadate $T$ : Lipopolysaccharide induces aberrant hypermethylation of Hic-1 in mouse embryonic fibroblasts lacking p53. Anticancer Res 28: 2101-2108, 2008

7. Kiefer JC: Epigenetics in development. Dev Dyn 236: 1144-1156, 2007.

8. Holliday R and Pugh JE: DNA modification mechanisms and gene activity during development. Science 187: 226-232, 1975.

9. Leonhardt $\mathrm{H}$ and Bestor TH: Structure, function and regulation of mammalian DNA methyltransferase. EXS 64: 109-119, 1993.

10. Klose RJ and Bird AP: Genomic DNA methylation: the mark and its mediators. Trends Biochem Sci 31: 89-97, 2006.

11. Heller G, Zielinski CC and Zöchbauer-Müller S: Lung cancer: from single-gene methylation to methylome profiling. Cancer Metastasis Rev 29: 95-107, 2010.

12. Adcock IM, Tsaprouni L, Bhavsar P and Ito K: Epigenetic regulation of airway inflammation. Curr Opin Immunol 19: 694-700, 2007.

13. Boellmann F, Zhang L, Clewell HJ, Schroth GP, Kenyon EM Andersen ME and Thomas RS: Genome-wide analysis of DNA methylation and gene expression changes in the mouse lung following subchronic arsenate exposure. Toxicol Sci 117: 404-417, 2010
14. Jia RZ, Zhang X, HU P, Liu XM, Hua XD, Wang X and Ding HJ: Screening for differential methylation status in human placenta in preeclampsia using a $\mathrm{CpG}$ island plus promoter microarray. Int J Mol Med 30: 133-141, 2012

15. Flores C, Pino-Yanes MM and Villar J: A quality assessment of genetic association studies supporting susceptibility and outcome in acute lung injury. Crit Care 12: R130, 2008.

16. Di Paola R, Cisafulli C, Mazzon E, Genovese T, Paterniti I, Bramanti P and Cuzzocrea S: Effect of PD98059, a selective MAPK3/MAPK1 inhibitor, on acute lung injury in mice. Int J Immunopathol Pharmacol 22: 937-950, 2009.

17. Grigoryev DN, Finigan JH, Hassoun P and Garcia JG: Science review: searching for gene candidates in acute lung injury. Crit Care 8: 440-447, 2004.

18. Yao HY, Chen L and Xu C: Inhibition of Rac activity alleviates lipopolysaccharide-induced acute pulmonary injury in mice. Biochim Biophys Acta 1810: 666-674, 2011.

19. Flores C, Pino-Yanes MM, Casula M, Casula M and Villar J: Genetics of acute lung injury: past, present and future. Minerva Anestesiol 76: 860-864, 2010.

20. Saxonov S, Berg P and Brutlag DL: A genome-wide analysis of $\mathrm{CpG}$ dinucleotides in the human genome distinguishes two distinct classes of promoters. Proc Natl Acad Sci USA 103: 1412-1417, 2006

21. Adamzik M, Frey U, Sixt S, Knemeyer L, Beiderlinden M, Peters J and Siffert W: ACE I/D but not AGT (-6)A/G polymorphism is a risk factor for mortality in ARDS. Eur Respir J 29: 482-488, 2007

22. Mura M, dos Santos CC, Stewart D and Liu M: Vascular endothelial growth factor and related molecules in acute lung injury. J Appl Physiol 97: 1605-1617, 2004.

23. Yan C, Wu M, Cao J, Tang H, Zhu M, Johnson PF and Gao H: Critical role for CCAAT/enhancer-binding protein $\beta$ in immune complex-induced acute lung injury. J Immunol 189: 1480-1490, 2012.

24. Belperio JA, Keane MP, Burdick MD, et al: Critical role for CXCR2 and CXCR2 ligands during the pathogenesis of ventilator-induced lung injury. J Clin Invest 110: 1703-1716, 2002.

25. Flores C, Ma SF, Maresso K, Wade MS, Villar J and Garcia JG: IL-6 gene-wide haplotype is association with susceptibility to acute lung injury. Transl Res 152: 11-17, 2008.

26. Han YJ, Ma SF, Wade MS, Flores C and Garcia JG: An intronic MYLK variant associated with inflammatory lung disease regulates promoter activity of the smooth muscle myosin light chain kinase isoform. J Mol (Berl) 90: 299-308, 2012.

27. Takahashi K, Koga K, Linge HM, et al: Macrophage CD74 contributes to MIF-induced pulmonary inflammation. Respir Res 10: 33, 2009.

28. Ikegami M, Falcone A and Whitsett JA: STAT-3 regulates surfactant phospholipid homeostasis in normal lung and during endotoxin-mediated lung injury. J Appl Physiol 104: 1753-1760, 2008.

29. Perl M, Chung CS, Perl U, Thakkar R, Lomas-Neira J and Ayala A: Therapeutic accessibility of caspase-mediated cell death as a key pathomechanism in indirect acute lung injury. Crit Care Med 38: 1179-1186, 2010.

30. Birukova AA, Xing J, Fu P, et al: Atrial natriuretic peptide attenuates LPS-induced vascular leak: role of PAK1. Am J Physiol Lung Cell Mol Physiol 299: L652-L663, 2010.

31. Leite-Junior JH, Garcia CS, Souza-Fernandes AB, et al: Methylprednisolone improves lung mechanics and reduces the inflammatory response in pulmonary but not in extrapulmonary mild acute lung injury in mice. Crit Care Med 36: 2621-2628, 2008. 